\title{
Survey: Improving Document Accessibility from the Blind and Visually Impaired User's Point of View
}

\author{
Martin Dorigo $^{1}$, Bettina Harriehausen-Mühlbauer ${ }^{2}$, Ingo Stengel ${ }^{2}$, \\ and Paul S. Dowland ${ }^{1}$ \\ ${ }^{1}$ Centre for Security, Communications and Network Research, \\ University of Plymouth, Plymouth, United Kingdom \\ ${ }^{2}$ University of Applied Sciences Darmstadt, Darmstadt, Germany \\ martin.dorigo@plymouth.ac.uk
}

\begin{abstract}
There are thousands of digital documents available on the internet, but many of them are not accessible for blind and visually impaired people. To find out what is of importance as to the reading of text and the navigation within documents from the user's point of view, a survey has been conducted among people concerned. They were asked how they handle text-documents, which problems they have and which features they would wish to have for a novel concept of assistive technology. The analysis of feedbacks from 205 participants leads to four key issues: The people concerned would like to have better access to digital documents as well as a better overview over their structure and content. In addition, the assistive technologies should be more easy, intuitive and standardised to use. A discussion is provided where the outline of preliminary solutions for the issues mentioned is proposed.
\end{abstract}

Keywords: Document accessibility, Visual impairment, Blind, Assistive Technology.

\section{Introduction}

\subsection{Essential Components}

On its way from the author to the reader with a disability, a digital document passes several components. To provide full access for the user, these components have to work together. If an accessibility feature is not implemented in one component, it does not result in an accessible user experience [1].

The author uses an authoring tool to create a document. The Authoring Tool Accessibility Guidelines (ATAG) [2] defines how authoring tools should help authors produce content that is accessible and conforms to the Web Content Accessibility Guidelines (WCAG) [3]. The content is typically read by a user agent. The User Agent Accessibility Guidelines (UAAG) [4] explains how to make user agents accessible to people with disabilities by passing the relevant information to the Accessibility API [5] of the operation system. This API helps assistive technologies like Screen Reader [6] to provide the information to the user with a disability. 


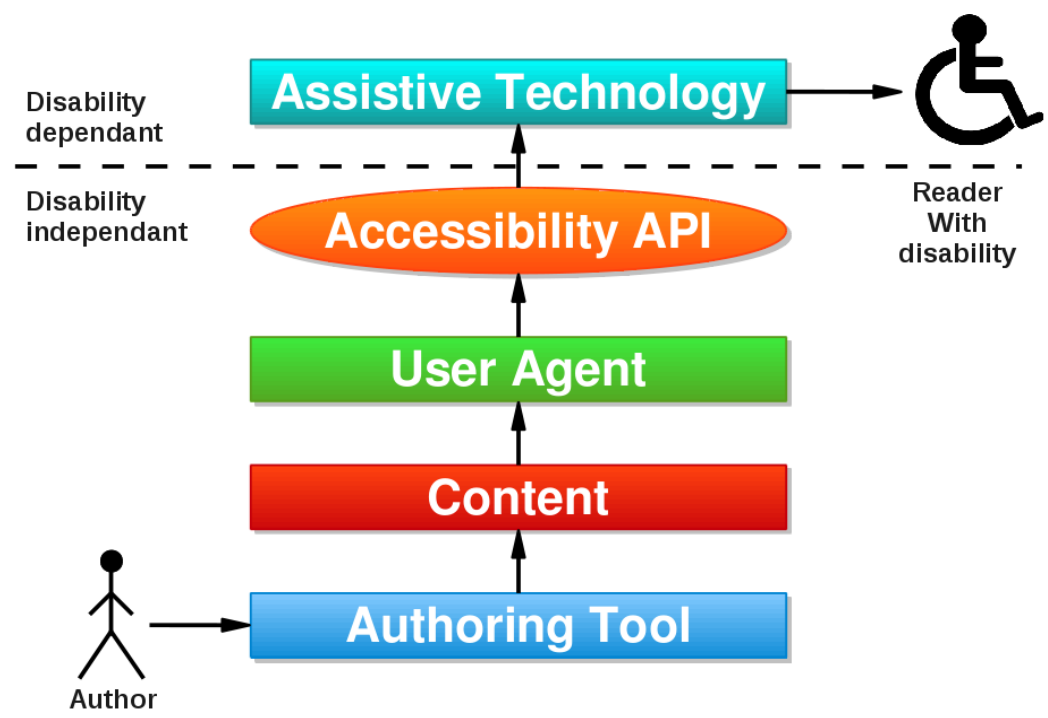

Fig. 1. Essential Components Overview

\subsection{Document Abstraction Layers}

During the processing by the different components, digital documents take on different forms. A static electronic document can be broken down into four abstraction layers [7].

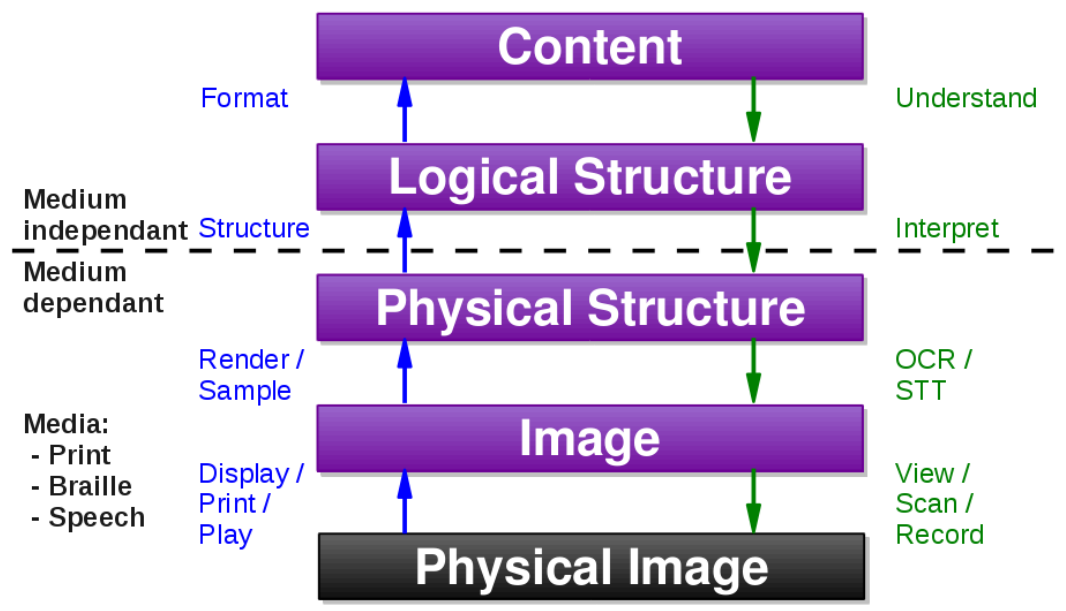

Fig. 2. Document Abstraction Layers 
After the documents content had been organized into a logical structure, it is formatted into the physical structure of a specific medium. In addition to print, other media relevant to blind and visually impaired are speech and braille. The physical structure can be rendered to an image, which is than displayed to the user by a specific physical device.

While the development can be easily done automatically by a machine, the document recognition on the other hand is very computationally intensive and defective. In order to make a document accessible to people with a disability, using different media, its logical structure must be available.

\section{Method}

\subsection{Participants}

$25^{`} 000$ blind and visually impaired people had been enquired in Austria, Germany and Switzerland. Feedbacks from 205 participants in the age from 18 to 80 years as well as in various occupational and educational situations were received. $55 \%$ male are accompanied by $45 \%$ female. The participants had been categorized into 6 categories of visual impairment according to the International Classification of Diseases (ICD) [8]. $27 \%$ of them are blind with no light perception, $22 \%$ with light perception and $16 \%$ with a visus worse than $0.05 .18 \%$ suffer from a severe visual impairment (visus worse than 0.1 ), $11 \%$ from a moderate visual impairment (visus worse than 0.3 ) and $6 \%$ from a mild visual impairment (visus worse than 1.0) [Fig. 3]. 61\% of them are visually impaired by birth.

$13 \%$ of all participants classified their computer literacy as little, $60 \%$ as good and $26 \%$ as very good [Fig. 4].

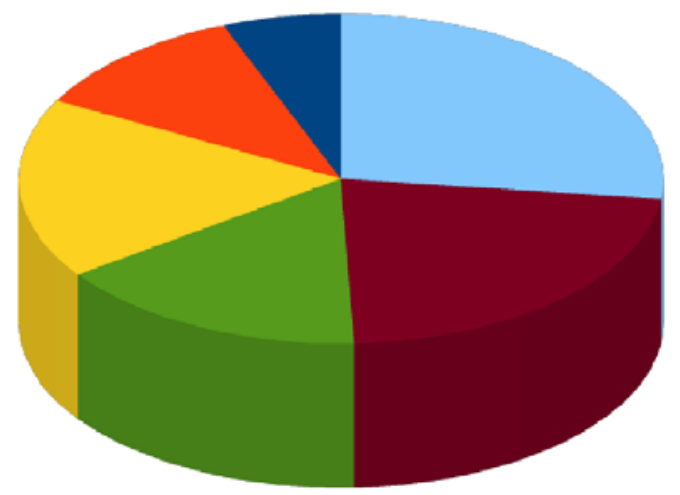

worse than $100 \%$ (mild visual pmpairment)

worse than $30 \%$ (moderate visual impairment)

worse than $10 \%$ (severe visual impairment)

worse than $5 \%$ (blind)

$1 \%$ (blind with light perception)

$0 \%$ (blind without light perception)

Fig. 3. Categories of visual impairment 


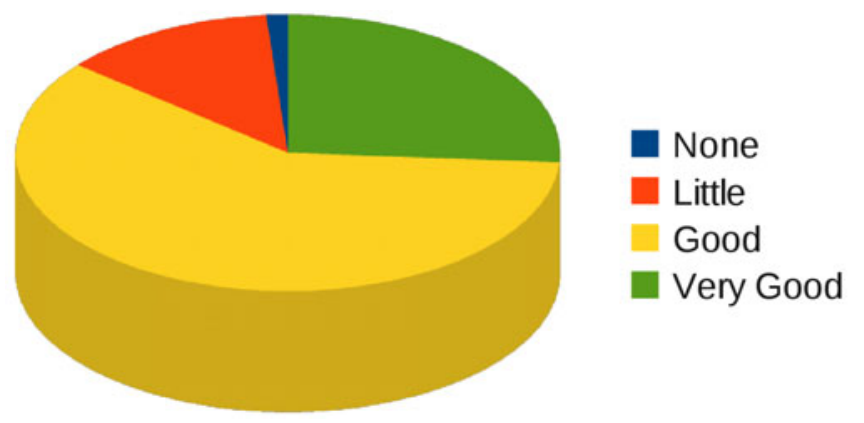

Fig. 4. Categories of computer literacy

\subsection{Questionnaire}

The questionnaire consists of 16 questions and covers the following areas of document accessibility. Most of the questions are multiple choices with a nominal or ordinal scale as well as a free text field for additional comments.

- Satisfaction

- Media and formats provided and wished

- Assistive technologies, input and other electronic devices in use

- Structural information

- Problems and solutions

- Wishes for a novel concept of an assistive technology

- Personal information: Visual impairment, occupational and educational situation and optionally contact details margin.

\subsection{Procedure}

Through organisations, online mailing lists, relevant forums and blogs as well as in paper based magazines blind and visually impaired people were invited to participate in this survey. In that invitation, the goal of the research was introduced to them. The participants could choose if they would like to take part online via the link provided or by phone in form of an interview. In the online survey, the quantitative aspect was promised while for people, questioned by phone, the qualitative aspect was centered. The answering took about 30 minutes. After the analysation of the results, four essential questions were resent to the 205 participants via email to get more detailed information.

\section{Results}

\subsection{Digital Formats}

The digital documents provided to blind and visually impaired people are a mix of different formats [Fig. 5]. The formats most used are HTML, Microsoft Word and PDF. 


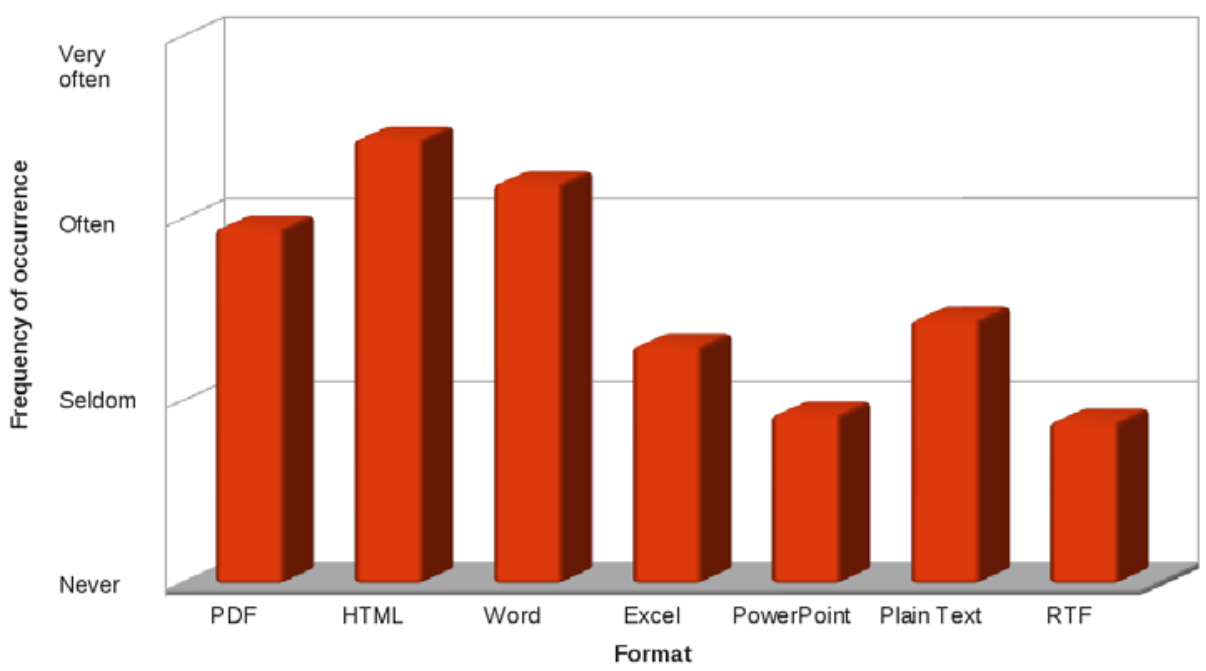

Fig. 5. Formats provided to blind and visually impaired

\subsection{Issues}

At the moment blind and visually impaired people are not very satisfied with their current situation as to the reading of text-documents. The problems and wishes mentioned lead to four key issues, where further research is needed.

Structure Overview. Speech can only provide one word at a given time. Braille can only show one line and people, working with heavy magnification can only see a single detail in a time. All of the participants wish a better overview over the structure

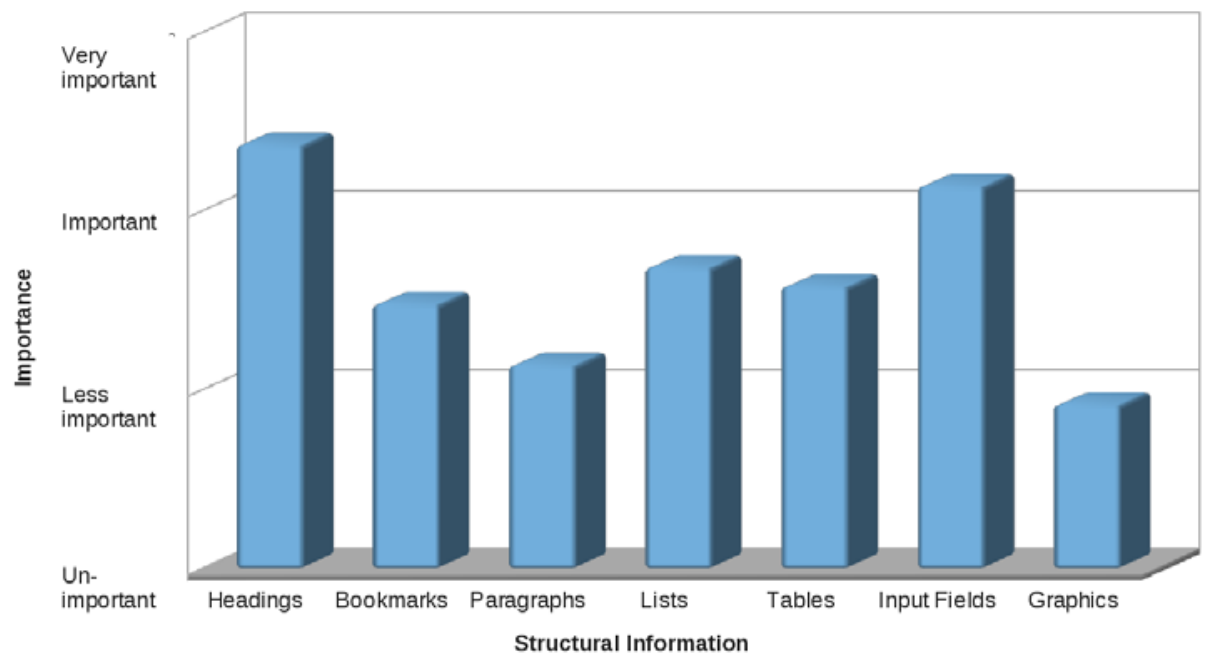

Fig. 6. Importance of Structural Informations 
of a document. The most important structural informations are headings and input fields followed by lists and tables. The least important element for blind and visually impaired is graphics [Fig. 6].

Content Overview. For blind and visually impaired people it is not possible to skim read a text passage to get a fast overview over the contained information. A faster overview over the document's content more than over the document's structure is wished.

Usability of Assistive Technologies. In order to read an digital document efficiently many key sequences have to be known by heart and they differentiate between specific products. An exception is Apple VoiceOver [9]. Especially people with little computer literacy wish that the assistive technology is more easy, intuitive and standardised to use.

Accessibility. All of the participants mentioned that they get documents which they cannot access. The concrete problems depend on the visual acuity a specific person comes with [Fig. 7].

All of the participants wish better accessibility of digital documents.

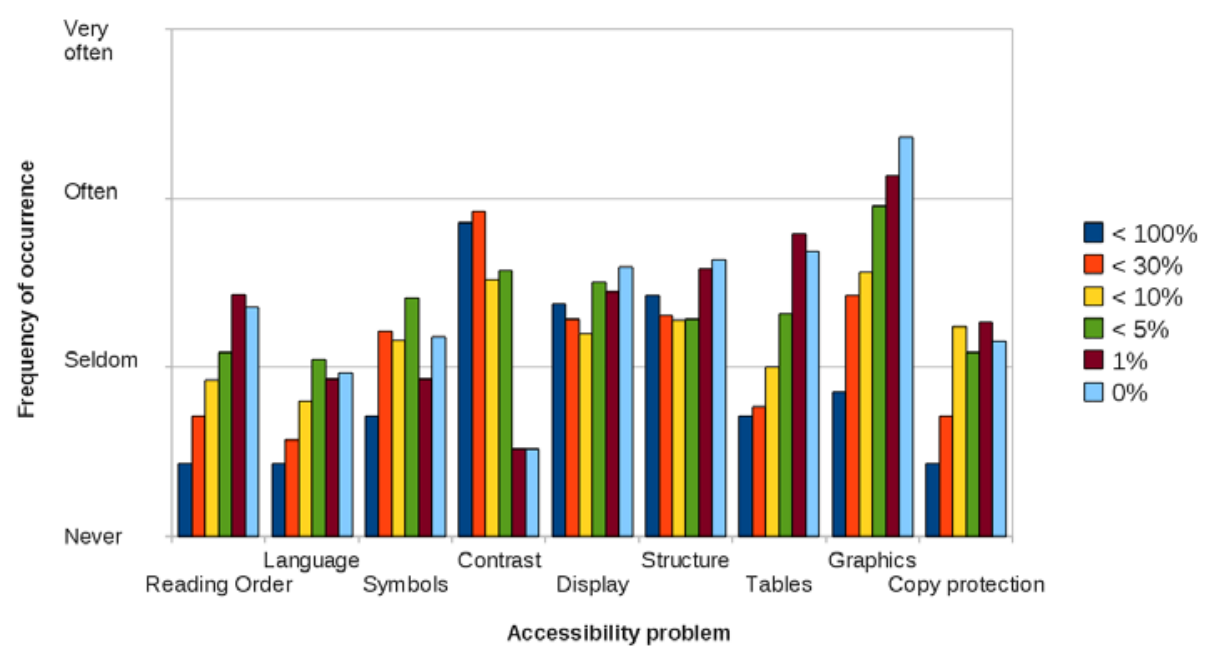

Fig. 7. Occurrence of accessibility problems

\section{Discussion and Further Work}

There is serious need for action. A concept should be made to display an overview over the document structure on other media such as speech and braille. In order to provide a content overview, the assistive technology needs comprehension over the document's content by using text mining. The usability may be improved by using alternative input devices like speech recognition or gestures. Accessibility is not a problem of missing theories rather than that they are not moved in the praxis. To improve this situation software may be developed which advises the author against potentially accessibility problems [10] or recognizes the document structure automatically [11]. 


\section{References}

1. W3C WAI Essential Components of Web Accessibility, http: / / www . w3 .org/WAI / intro/ components . php

2. W3C WAI Authoring Tool Accessibility Guidelines (ATAG) 2.0, http: / / www.w3 .org/TR/ATAG20/

3. W3C WAI Web Content Accessibility Guidelines (WCAG) 2.0, http: / / www.w3 .org/TR/WCAG2 0 /

4. W3C WAI User Agent Accessibility Guidelines (UAAG) 2.0, http: / / www .w3 . org/TR/UAAG2 0/

5. Microsoft Active Accessibility (MSAA), http://msdn.microsoft.com/ en-us / library / dd373592\%28VS . 85\%29. aspx

6. Freedom Scientific - JAWS for Windows Screen Reading Software, http: / /www. freedomscientific.com/products/fs / jaws-product-page.asp

7. Brugger, R.: Eine statistische Methode zur Erkennung von Dokumentstrukturen. PhD thesis no. 1251, University of Fribourg (1998)

8. WHO International Classification of Diseases (ICD), http: / / www . who.int/classifications/icd/en/

9. Apple Accessibility VoiceOver, http: / /www.apple.com/accessibility/voiceover/

10. Davishy, A., Hutter, H.P., Horvath, A., Dorigo, M.: A Flexible Software Architecture Concept for the Creation of Accessible PDF Documents. In: Miesenberger, K., Klaus, J., Zagler, W., Karshmer, A. (eds.) ICCHP 2010. LNCS, vol. 6179, pp. 47-52. Springer, Heidelberg (2010)

11. Ruemer, R., Miesenberger, K., Kummer, F., Gravenhorst, C.: Improving the Redigitisation Process by Using Software with Automatic Metadata Detection. In: Miesenberger, K., Klaus, J., Zagler, W., Karshmer, A. (eds.) ICCHP 2010. LNCS, vol. 6179, pp. 35-42. Springer, Heidelberg (2010) 